Article

\title{
Improved Subtyping of Avian Influenza Viruses Using an RT-qPCR-Based Low Density Array: 'Riems Influenza a Typing Array', Version 2 (RITA-2)
}

\author{
Kareem E. Hassan 1,2 ${ }^{\mathbb{D}}$, Ann Kathrin Ahrens ${ }^{1}$, Ahmed Ali ${ }^{2} \mathbb{D}$, Magdy F. El-Kady ${ }^{2}$, Hafez M. Hafez ${ }^{3}$, \\ Thomas C. Mettenleiter ${ }^{4}\left(\mathbb{D}\right.$, Martin Beer ${ }^{1}$ and Timm Harder ${ }^{1, *(D)}$ \\ 1 Institute of Diagnostic Virology, Friedrich-Loeffler-Institut, 17493 Greifswald-Insel Riems, Germany; \\ kareem_eid@yahoo.com (K.E.H.); annkathrin.ahrens@fli.de (A.K.A.); martin.beer@fli.de (M.B.) \\ 2 Department of Poultry Diseases, Faculty of Veterinary Medicine, Beni-Suef University, Beni-Suef 62511, Egypt \\ ali.178w@yahoo.com (A.A.); mfelkady@yahoo.com (M.F.E.-K.) \\ 3 Institute of Poultry Diseases, Free University Berlin, 14163 Berlin, Germany; hafez.mohamed@fu-berlin.de \\ 4 Friedrich-Loeffler-Institute, 17493 Greifswald-Insel Riems, Germany; thomas.mettenleiter@fli.de \\ * Correspondence: timm.harder@fli.de; Tel.: +49-(0)-3-8351-71546
}

check for

updates

Citation: Hassan, K.E.; Ahrens, A.K.; Ali, A.; El-Kady, M.F.; Hafez, H.M.; Mettenleiter, T.C.; Beer, M.; Harder, T. Improved Subtyping of Avian Influenza Viruses Using an RT-qPCR-Based Low Density Array: 'Riems Influenza a Typing Array', Version 2 (RITA-2). Viruses 2022, 14, 415. https://doi.org/10.3390/ v14020415

Academic Editor: Feng Li

Received: 29 December 2021

Accepted: 15 February 2022

Published: 17 February 2022

Publisher's Note: MDPI stays neutral with regard to jurisdictional claims in published maps and institutional affiliations.

Copyright: (C) 2022 by the authors. Licensee MDPI, Basel, Switzerland. This article is an open access article distributed under the terms and conditions of the Creative Commons Attribution (CC BY) license (https:// creativecommons.org/licenses/by/ $4.0 /)$.

\begin{abstract}
Avian influenza virus (AIV) variants emerge frequently, which challenges rapid diagnosis. Appropriate diagnosis reaching the sub- and pathotype level is the basis of combatting notifiable AIV infections. Real-time RT-PCR (RT-qPCR) has become a standard diagnostic tool. Here, a total of 24 arrayed RT-qPCRs is introduced for full subtyping of 16 hemagglutinin and nine neuraminidase subtypes of AIV. This array, designated Riems Influenza A Typing Array version 2 (RITA-2), represents an updated and economized version of the RITA-1 array previously published by Hoffmann et al. RITA-2 provides improved integration of assays (24 instead of 32 parallel reactions) and reduced assay volume $(12.5 \mu \mathrm{L})$. The technique also adds RT-qPCRs to detect Newcastle Disease (NDV) and Infectious Bronchitis viruses (IBV). In addition, it maximizes inclusivity (all sequences within one subtype) and exclusivity (no intersubtypic cross-reactions) as shown in validation runs using a panel of 428 AIV reference isolates, 15 reference samples each of NDV and IBV, and 122 clinical samples. The open format of RITA-2 is particularly tailored to subtyping influenza A virus of avian hosts and Eurasian geographic origin. Decoupling and re-arranging selected RT-qPCRs to detect specific AIV variants causing epizootic outbreaks with a temporal and/or geographic restriction is possible.
\end{abstract}

Keywords: avian influenza; diagnosis; real-time RT-PCR; Newcastle disease virus; infectious bronchitis virus

\section{Introduction}

Highly pathogenic avian influenza virus (HPAIV) is the major pathogen associated with substantial economic losses in poultry production. Zoonotic AIV strains, in addition, have caused multiple cases of human infections, sparking influenza pandemic concerns [1]. The influenza A virus genome consists of eight single-stranded RNA gene segments. Two segments encode the major envelope glycoproteins species of these viruses, hemagglutinin (HA) and neuraminidase (NA). These proteins have essential functions in defining host and tissue tropism and influence virulence. The HA, in particular, is a main target of the protective humoral immune response. Based on nucleotide sequence and protein antigenicity of the HA and NA surface glycoproteins, AIVs are classified into 16 different HA (H1-H16) and 9 NA subtypes (N1-N9) [2,3]. The distinctive segmental structure of influenza virus genomes enables reassortment of segments if the same host cell is infected by two (or more) different parental viruses. Theoretically, 144 combinations between HA and NA may result. However, not all of these have actually been detected in nature as there seem to be predilections of certain HA and NA combinations [4]. Moreover, there 
is a continuous turnover, with temporal and geographical restrictions, of different AIV subtypes and their variants in the reservoir hosts as in aquatic wild bird populations [5]. Spill-over infections into poultry populations, often starting endemic transmission chains with similar reassortment events, widen the replication basis of these viruses. Error-prone viral genome replication drives genetic drift and further increases genetic and antigenic variability [1,4]. In summary, these processes present a continuous challenge not only for the immune system of the avian hosts but also for accurate and rapid laboratory diagnosis.

Swift and exact diagnosis of AIV infections in poultry populations is pivotal to inform veterinary authorities and steer restriction measures if notifiable AIV subtypes, i.e., H5 and $\mathrm{H7}$, are detected. Adequate control of zoonotic AIV infections in poultry populations is also the most important measure to limit exposure of human populations to these viruses [6]. Aquatic wild birds play a major role in the evolution, maintenance, and spread of AIV. Therefore, optimized surveillance of reservoir populations is important to follow viral evolutionary trajectories [7]. Conventional techniques for AIV diagnosis include virus isolation in embryonated chicken eggs, serological characterization of virus isolates, and animal experiments to define viral pathogenicity [4]. Time until final diagnosis using these methods may take up to two weeks. Minimizing time until diagnosis, therefore, is the main objective of new diagnostic developments. Rapid antigen detection assays based on lateral flow immunochromatography were particularly successful in this respect but lacked sensitivity [8-10] when compared to reverse transcriptase-polymerase chain reaction (RT-PCR) and especially time-saving real-time RT-PCR technologies (RT-qPCR). Thus, RT-qPCRs have become new standards [11-15]. These include RT-qPCRs for generic AIV detection, subtyping, and pathotyping where the latter are targeting the HA cleavage site [16-18].

In 2016, Hoffmann et al. [16] published an assay assembling several subtype-specific RT-qPCRs into a low-density PCR array, designated as the 'Riems Influenza A Typing Array' (RITA). RITA enabled AIV RNA detection at subtype level in clinical samples by providing a generic, internally controlled M gene-specific duplex RT-qPCR and a further 31 monoplex TaqMan ${ }^{\circledR}$-based RT-qPCRs to differentiate 14 HA and nine NA subtypes. Although RITA proved suitable for use in routine diagnostic applications, shortcomings in terms of minor cross-reactivities between closely related subtypes (e.g., H2/H5, H7/H10/H15, H1/H6) were noted. These effects caused subtle problems in ruling out co-infections with several AIV subtypes. In addition, the demand to performing in parallel 32 single RT-qPCRs might have a repelling effect on potential users.

In this study, an updated and improved version of RITA (syn. RITA-2) is developed and validated. The new version considers grossly enlarged databases of Eurasian AIV sequences since 2015, when the previous RITA version was designed. Intersubtypic crossreactions have been abrogated by re-designing primers and probes so as to select target locations that were less conserved between the subtypes. Many primers and probes have been newly established so as to also meet the growing demands for full inclusivity of all Eurasian virus sequences of a certain subtype available in public databases. The revised version also achieved a higher degree of assay integration by using multiplexing of RT-qPCRs more stringently. Space on the array was economized to accommodate four instead of three clinical samples per 96-well array plate and to include all $16 \mathrm{HA}$ and nine NA AIV subtypes in addition to an influenza A generic RT-qPCR. Also, targets for other important avian pathogens, Newcastle Disease virus (NDV) and Infectious Bronchitis virus (IBV), have been added to the design for differential diagnostic means. In addition, we show how the RITA-2 array can be used as an "assay mine" and single reactions be decoupled and recombined into much smaller arrays tailored to routine diagnosis during HPAIV epizootics. 


\section{Materials and Methods}

\subsection{Viruses}

RNA extracted from 428 influenza viral strains representing $16 \mathrm{HA}$ and nine NA subtypes pre-typed (either serologically or by sequencing) at the National Reference Laboratory for Avian Influenza, Friedrich-Loeffler-Institut (NRL-AI, FLI, Isle of Riems, Germany) were used for analytical validation of the newly developed assays. Table 1 provides a condensed overview of the subtypes used and their species of origin. In addition, fifteen reference isolates each for IB and ND viruses were used for re-evaluation of previously established IBV and NDV RT-qPCR assays (Table S1).

Table 1. Collection of influenza A virus strains of different host origin and differing subtypes (hemagglutinin, HA, and neuraminidase, NA) used for evaluation of real-time RT-PCRs.

\begin{tabular}{|c|c|c|c|c|c|c|}
\hline \multirow{2}{*}{ Subtype } & \multirow{2}{*}{$\begin{array}{l}\text { Number of } \\
\text { Samples }\end{array}$} & \multicolumn{5}{|c|}{ Host } \\
\hline & & Avian & Human & Porcine & Equine & Unknown \\
\hline H1 & 63 & 10 & 17 & 32 & & 4 \\
\hline H2 & 20 & 19 & 1 & & & \\
\hline H3 & 52 & 25 & 11 & 15 & 1 & \\
\hline H4 & 20 & 20 & & & & \\
\hline H5 & 53 & 53 & & & & \\
\hline H6 & 67 & 67 & & & & \\
\hline H7 & 42 & 40 & & & 1 & 1 \\
\hline H8 & 5 & 5 & & & & \\
\hline H9 & 43 & 29 & & & & 14 \\
\hline H10 & 32 & 30 & & 2 & & \\
\hline H11 & 14 & 12 & & & 1 & 1 \\
\hline H12 & 1 & 1 & & & & \\
\hline H13 & 10 & 10 & & & & \\
\hline H14 & 1 & 1 & & & & \\
\hline H15 & 1 & 1 & & & & \\
\hline H16 & 4 & 4 & & & & \\
\hline HA, total & 428 & 327 & 29 & 49 & 3 & 20 \\
\hline N1 & 144 & 93 & 17 & 30 & & 4 \\
\hline N2 & 125 & 88 & 11 & 12 & & 14 \\
\hline N3 & 29 & 29 & & & & \\
\hline $\mathrm{N} 4$ & 10 & 10 & & & & \\
\hline N5 & 6 & 5 & & & & 1 \\
\hline N6 & 28 & 23 & & 4 & & 1 \\
\hline N7 & 38 & 34 & & 3 & 1 & \\
\hline N8 & 37 & 36 & & & 1 & \\
\hline N9 & 11 & 9 & 1 & & 1 & \\
\hline NA, total & 428 & 327 & 29 & 49 & 3 & 20 \\
\hline
\end{tabular}

\subsection{Clinical Material}

Oropharyngeal and cloacal swabs collected from diseased or freshly dead birds were submitted to the NRL-AI. Egyptian samples consisted of pools of 15 to 20 swabs from each flock of poultry and were collected from duck, chicken, and turkey farms in Egypt during 
2018 and from Egyptian chicken flocks during early 2019 (47 samples in total). Samples were suspended in $2 \mathrm{~mL}$ of phosphate-buffered saline (PBS), pH 7.0-7.4, and clarified by centrifugation at $4000 \mathrm{rpm}$ for $10 \mathrm{~min}$ at the Department of Poultry Diseases (Beni-Suef University, Beni Suef, Egypt). About $200 \mu \mathrm{L}$ of clarified supernatant were uploaded on Whatman ${ }^{\circledR}$ FTA ${ }^{\circledR}$ Cards (Merck, Darmstadt, Germany) and sent to NRL-AI at FLI, Germany. In addition, oro-nasal and cloacal swabs collected from clinically healthy duck flocks from Bangladesh during 2019 were included (13 samples in total) as well as routine diagnostic samples submitted for diagnosis during HPAI epizootics in Germany ( $n=62$; Table S2).

\subsection{Primers and Probes}

Primers and probes reported for version 1 of the RITA array were double-checked against the EpiFlu database of GISAID (http://platform.gisaid.org), the International Nucleotide Sequence Data Collaboration IRD (http:/ / www.fludb.org) sequence databases or against comprehensive alignments of HA and NA sequences built from these. Sequences were handled using the Geneious software, version 11.1.7 [19]. Alignment and identity matrix analyses were generated using MAFFT [20] and manually edited with AliView [21]. The focus of searches and comparisons was on sequences added to the databases since 2015. The chemical properties of preselected oligos were analyzed by the OligoCalc software [22]. Oligos were synthesized by Metabion GmbH (Martinsried, Germany) and Eurogentec (Liège, Belgium). Oligos were solubilized to produce stock solutions of $100 \mathrm{pmol} \mu \mathrm{L}^{-1}$ and stored at $-20^{\circ} \mathrm{C}$ until use. For use in PCRs, stock solutions were diluted to give a final



Table 2. The final design of primers and probes used for assembling the RITA-2 array.

\begin{tabular}{|c|c|c|c|c|}
\hline Subtype & Designation & Sequence $5^{\prime} \Rightarrow 3^{\prime}$ & Amount $^{1}$ & Reference \\
\hline \multirow{5}{*}{ Pan AI assay } & M1-F & AGA TGA GYC TTC TAA CCG AGG TCG & $20.0 \mu \mathrm{L}$ & \multirow{5}{*}[4,15]{} \\
\hline & M1-FAM & FAM-TCA GGC CCC CTC AAA GCC GA-BHQ1 & $2.5 \mu \mathrm{L}$ & \\
\hline & M1-R1 & TGC AAA AAC ATC TTC AAG TYT CTG & $15.0 \mu \mathrm{L}$ & \\
\hline & M1-R2 & TGC AAA GAC ACT TTC CAG TCT CTG & $15.0 \mu \mathrm{L}$ & \\
\hline & M1-R3 & TGC AAA I(Inosine)AC ATC YTC AAG TYT CTG & $7 \mu \mathrm{L}$ & \\
\hline \multirow{6}{*}{ H1 assay } & H1-F1 & CCA TCT GTA TAG GCT AYC AT & $20 \mu \mathrm{L}$ & \multirow{6}{*}{ This study $^{2}$} \\
\hline & H1-F2 & AAA CAT YCC TTC CRT TCA ATC & $20 \mu \mathrm{L}$ & \\
\hline & H1-FAM1 & FAM-TAC AGA CAC TGT YGA CAC DGT GCT-BHQ1 & $5 \mu \mathrm{L}$ & \\
\hline & H1-FAM2 & FAM-TTC ATT GAA GGR GGR TGG ACA GGA AT-BHQ1 & $5 \mu \mathrm{L}$ & \\
\hline & H1-R1 & GTG AGT CAC RGT YAC ATT CTT & $20 \mu \mathrm{L}$ & \\
\hline & H1-R2 & GAG CAA GGI TCY GGT TAT G & $20 \mu \mathrm{L}$ & \\
\hline \multirow{3}{*}{ H2 assay } & H2-F & CTA AST GTR CCW GAA TGG TC & $40 \mu \mathrm{L}$ & \multirow{3}{*}{ This study ${ }^{2}$} \\
\hline & H2-R & GAG GTG TTT CAR TTC YTC RTA & $40 \mu \mathrm{L}$ & \\
\hline & H2-FAM & FAM-TGT GCT ACC CAG GYA GTT TCA ATG A -BHQ1 & $8 \mu \mathrm{L}$ & \\
\hline \multirow{9}{*}{ H3 assay } & H3-F1 & CCT CGR GGC TAY TTC AAR AT & $15 \mu \mathrm{L}$ & \multirow{9}{*}{ This study } \\
\hline & H3-F2 & AGA CTG GAT CYT RTG GAT TTC & $15 \mu \mathrm{L}$ & \\
\hline & H3-F3 & CTG GGR CAC CAT GCA GT & $15 \mu \mathrm{L}$ & \\
\hline & H3-FAM1 & FAM-TGC ATC TGA YCT CAT TAT YGA RCT TTT-BHQ1 & $4 \mu \mathrm{L}$ & \\
\hline & H3-FAM2 & FAM-ACR CAA AGC AAA AAG CAT GAT ATG GC-BHQ1 & $4 \mu \mathrm{L}$ & \\
\hline & H3-FAM3 & FAM-ACA GGG AAA ATA TGC ARC AAT CCY CA-BHQ1 & $4 \mu \mathrm{L}$ & \\
\hline & H3-R1 & ATT TGG RGT GAT RCA TTC AGA & $15 \mu \mathrm{L}$ & \\
\hline & H3-R2 & CTC AAA TGC AAA TGK TGC AYC & $15 \mu \mathrm{L}$ & \\
\hline & H3-R3 & TGT GCA GTC YCT TCC ATC & $15 \mu \mathrm{L}$ & \\
\hline \multirow{4}{*}{ H4 assay } & H4-F1 & ACYCAGGGRTACAAGGACA & $20 \mu \mathrm{L}$ & \multirow{4}{*}{ This study ${ }^{2}$} \\
\hline & H4-F2 & GGA CAT CAT YCT YTG GAT TTC & $20 \mu \mathrm{L}$ & \\
\hline & H4-FAM & FAM-TCC ATA TCA TGC TTY TTG CTY GTA GC-BHQ1 & $4 \mu \mathrm{L}$ & \\
\hline & H4-R & CAA GCC CAC AAA AYR AAG G & $40 \mu \mathrm{L}$ & \\
\hline
\end{tabular}


Table 2. Cont.

\begin{tabular}{|c|c|c|c|c|}
\hline Subtype & Designation & Sequence $5^{\prime} \Rightarrow 3^{\prime}$ & Amount ${ }^{1}$ & Reference \\
\hline \multirow{7}{*}{ H5 assay } & H5-HA1-F & GAT TYT AAA RGA TTG TAG YGT AGC & $20 \mu \mathrm{L}$ & \multirow{7}{*}{ This study ${ }^{2}$} \\
\hline & H5-FAM3-RC & FAM-CGC ACA TTG GRT TYC CRA GGA GCC-BHQ1 & $6 \mu \mathrm{L}$ & \\
\hline & H5-HA1-R1 & CTC TCY ACC ATG TAR GAC CA & $15 \mu \mathrm{L}$ & \\
\hline & H5-HA1-R2 & CTC TCY ACT ATG TAR GAC CA & $15 \mu \mathrm{L}$ & \\
\hline & H5-F2 & GTT CCC TAG YAY TGG CAA TCA T & $20 \mu \mathrm{L}$ & \\
\hline & H5-FAM2 & FAM-CTG GTC TAT YYT TRT GGA TGT GCT CC-BHQ1 & $6 \mu \mathrm{L}$ & \\
\hline & H5-R2 & AAT TCT ARA TGC AAA TTC TGC AYT G & $15 \mu \mathrm{L}$ & \\
\hline \multirow{4}{*}{ H6 assay } & H6-F1 & TTG GYG TGT ATC AAA TYC TTK C & $20 \mu \mathrm{L}$ & \multirow{4}{*}{ This study ${ }^{2}$} \\
\hline & H6-F2 & TTG RCG TGT ATC AAA TAC TTG C & $20 \mu \mathrm{L}$ & \\
\hline & H6-FAM & FAM-AGR CTG CTC GAY ACC GTA CTA TAA A-BHQ1 & $10 \mu \mathrm{L}$ & \\
\hline & H6-R & TTGA RCY ATT TGA ACA CAT CCA & $40 \mu \mathrm{L}$ & \\
\hline \multirow{5}{*}{ H7 assay } & H7-F & CAA CTG AAA CRG TRG ARC G & $45 \mu \mathrm{L}$ & \multirow{5}{*}{ This study ${ }^{2}$} \\
\hline & H7-FAM & FAM-CCC AGG ATY TGC TCA ARA GGR AAA A-BHQ1 & $10 \mu \mathrm{L}$ & \\
\hline & H7-R1 & CAG GAG YCC ACA TTG ACC & $15 \mu \mathrm{L}$ & \\
\hline & H7-R2 & CAG WAG YCC ACA TTG ACC & $15 \mu \mathrm{L}$ & \\
\hline & H7-R3 & TTC TAG GAA TTG GTC ACA TTG & $15 \mu \mathrm{L}$ & \\
\hline \multirow{3}{*}{ H8 assay } & H8-F & CCA CCT AYA AAA TTC TCA GCA & $40 \mu \mathrm{L}$ & This study ${ }^{2}$ \\
\hline & H8-FAM & FAM-TGC CAA GCA RAG ACT GGC CGC CA-BHQ1 & $4 \mu \mathrm{L}$ & [16] \\
\hline & H8-R & ARA CCT CCA GCA AYC AGG A & $40 \mu \mathrm{L}$ & This study ${ }^{2}$ \\
\hline \multirow{4}{*}{ H9 assay } & H9-F1 & CAA TGG GGT TYG CTG CCT & $20 \mu \mathrm{L}$ & \multirow{4}{*}{ [23] } \\
\hline & H9-F2 & CAA TGG GRK TTG CTG CCT & $20 \mu \mathrm{L}$ & \\
\hline & H9-FAM & FAM-TTY TGG GCC ATG TCI AAT GGR TC-BHQ1 & $6 \mu \mathrm{L}$ & \\
\hline & H9-R & TTA TAT ACA RAT GTT GCA YCT G & $40 \mu \mathrm{L}$ & \\
\hline \multirow{3}{*}{ H10 assay } & H10-F & CAA CTC AGR CAG AAT GCW GA & $40 \mu \mathrm{L}$ & \multirow{3}{*}{ This study ${ }^{2}$} \\
\hline & H10-FAM & FAM-TGC ATG GAG AGY ATA AGR AAC AAC AC-BHQ1 & $6 \mu \mathrm{L}$ & \\
\hline & H10-R & CTT CYT CTC TGT AYT GTG AAT G & $40 \mu \mathrm{L}$ & \\
\hline \multirow{3}{*}{ H11 assay } & H11-F & GGA CAT ATG AYC ACA ARG AAT T & $40 \mu \mathrm{L}$ & \multirow{3}{*}{ This study ${ }^{2}$} \\
\hline & H11-FAM & FAM-ACT GTC RAT TTA CAG CTG CAT YGC A-BHQ1 & $8 \mu \mathrm{L}$ & \\
\hline & H11-R & ATG CAA ATG GTA CAT CTA CAT G & $40 \mu \mathrm{L}$ & \\
\hline \multirow{3}{*}{ H12 assay } & H12-F & CAT CTA CAG CAG YGT YGC & $40 \mu \mathrm{L}$ & \multirow{3}{*}{ This study ${ }^{2}$} \\
\hline & H12-FAM & FAM-ACT GCT CAT GAT TAT TGG GGG TTT CA-BHQ1 & $12 \mu \mathrm{L}$ & \\
\hline & H12-R & GAA AGT ACA ACG AAC ATT TCC A & $40 \mu \mathrm{L}$ & \\
\hline \multirow{7}{*}{ H13 assay } & H13-F1 & CTT AAG CAC AAA CTC ATC AGA A & $15 \mu \mathrm{L}$ & \multirow{7}{*}{ This study } \\
\hline & H13-F2 & CTG AGC ACC AAT TCA TCA GA & $15 \mu \mathrm{L}$ & \\
\hline & H13-F3 & CTT AAG CAC AAA CTC ATC AGA A & $15 \mu \mathrm{L}$ & \\
\hline & H13-FAM1 & FAM-CKA ACC ACA CRG GAA CAT AYT GTT C-BHQ1 & $5 \mu \mathrm{L}$ & \\
\hline & H13-FAM2 & FAM-CAC ACI GGA ACA TWC TGT TCA ATC A-BHQ1 & $5 \mu \mathrm{L}$ & \\
\hline & H13-R1 & CTG GCA CAG GCA GGG TT & $20 \mu \mathrm{L}$ & \\
\hline & H13-R2 & CCY ACA ATC CAT CCT TCA AA & $20 \mu \mathrm{L}$ & \\
\hline \multirow{3}{*}{ H14 assay } & H14-F & CCC AAT ATA GGA AGT AGA CC & $40 \mu \mathrm{L}$ & \multirow{3}{*}{ This study ${ }^{2}$} \\
\hline & H14-HEX & HEX-AAG CAT CTA CTG GAC YCT AGT AAA CC-BHQ1 & $6 \mu \mathrm{L}$ & \\
\hline & H14-R & CTT CTT GTC ACT TYT AAG CAC & $40 \mu \mathrm{L}$ & \\
\hline \multirow{3}{*}{ H15 assay } & H15-F & CAS CTT TCT CCG CTC TAA TG & $40 \mu \mathrm{L}$ & \multirow{3}{*}{ This study ${ }^{2}$} \\
\hline & H15-FAM & FAM-CAC TGG GAA TAC AGA GTG ATG CAC AA-BHQ1 & $3 \mu \mathrm{L}$ & \\
\hline & H15-R & AAR CAT TCC CCT TCA CAT GA & $40 \mu \mathrm{L}$ & \\
\hline \multirow{3}{*}{ H16 assay } & H16-F & ARY TGA AGA CTG AAG ACA ATG T & $40 \mu \mathrm{L}$ & \\
\hline & H16-HEX & HEX-CTG GTA GGW CTC ATA CTY GCA TTT AT-BHQ1 & $6 \mu \mathrm{L}$ & This study ${ }^{2}$ \\
\hline & H16-R & CCA CTG CTG CAT GCC CA & $40 \mu \mathrm{L}$ & \\
\hline
\end{tabular}


Table 2. Cont.

\begin{tabular}{|c|c|c|c|c|}
\hline Subtype & Designation & Sequence $5^{\prime} \Rightarrow 3^{\prime}$ & Amount $^{1}$ & Reference \\
\hline \multirow{3}{*}{ N1 assay } & N1-F & GRC CTT GYT TCT GGG TKG A & $40 \mu \mathrm{L}$ & \multirow{2}{*}{ This study ${ }^{2}$} \\
\hline & N1-FAM & FAM-CAA TYT GGA CYA GTG GRA GYA GCA T-BHQ1 & $6 \mu \mathrm{L}$ & \\
\hline & N1-R & ACC GTC TGG CCA AGA CCA & $40 \mu \mathrm{L}$ & [16] \\
\hline \multirow{4}{*}{ N2 assay } & N2-F1 & AGTC TGG TGG ACY TCA AAY AG & $20 \mu \mathrm{L}$ & [16] \\
\hline & N2-F2 & CAG AGT RTG GTG GAC ITC & $20 \mu \mathrm{L}$ & \multirow{3}{*}{ [23] } \\
\hline & N2-FAM & FAM-CAT CAG GCC ATG AGC CTG TYC CAT-BHQ1 & $4 \mu \mathrm{L}$ & \\
\hline & N2-R & TTG CGA AAG CTT AYA TNG VCA T & $40 \mu \mathrm{L}$ & \\
\hline \multirow{4}{*}{ N3 assay } & N3-F & GCA AYA GTA TAG TTA CYT TCT G & $40 \mu \mathrm{L}$ & \multirow{4}{*}{ This study ${ }^{2}$} \\
\hline & N3-FAM & FAM-AGA CAA TGA ACC TGG ATC GGG VAA-BHQ1 & $3 \mu \mathrm{L}$ & \\
\hline & N3-R1 & TTA CTT GGG CAT RAA CCC AAT & $20 \mu \mathrm{L}$ & \\
\hline & N3-R2 & GTT GGM ACC RTC WGG CCA & $20 \mu \mathrm{L}$ & \\
\hline \multirow{4}{*}{ N4 assay } & N4-F1 & GAC TAG YGG TAG TAG YAT TGC & $20 \mu \mathrm{L}$ & This study ${ }^{2}$ \\
\hline & N4-F2 & AGT AGY ATT GCR TTY TGT GGT GTT & $20 \mu \mathrm{L}$ & \multirow{2}{*}{ [16] } \\
\hline & N4-HEX & HEX-TGG TCR TGG CCY GAT GGC GCT CT-BHQ1 & $6 \mu \mathrm{L}$ & \\
\hline & N4-R & CGA AAA ATY ACT TGT CTA TGT CAA & $40 \mu \mathrm{L}$ & This study ${ }^{2}$ \\
\hline \multirow{4}{*}{ N5 assay } & N5-F1 & CCT TCA GAA TGC AGR ACY TT & $20 \mu \mathrm{L}$ & \multirow{4}{*}{ This study ${ }^{2}$} \\
\hline & N5-F2 & CAA ATA ATA CAG TAA ARG ACA GAA G & $20 \mu \mathrm{L}$ & \\
\hline & N5-HEX & HEX-TAA TGA GCG TRC CAT TGG GAT CCT C-BHQ1 & $6 \mu \mathrm{L}$ & \\
\hline & N5-RR & TAG CAG ACC AYC CRA CGG A & $40 \mu \mathrm{L}$ & \\
\hline \multirow{7}{*}{ N6 assay } & N6-F1 & GGT GAM AAT GAA YCC AAA YCA & $15 \mu \mathrm{L}$ & \multirow{2}{*}{ [16] } \\
\hline & N6-F2 & AAT GAA YCC AAA YCA RAA GAT AA & $15 \mu \mathrm{L}$ & \\
\hline & N6-F3 & GAA AAT GAA TCC AAA TCA RAA GRT A & $15 \mu \mathrm{L}$ & \multirow{8}{*}{ This study $^{2}$} \\
\hline & N6-FAM & FAM-CAT YTC AGC IAG GAR TRA CAC TAT C-BHQ1 & $12 \mu \mathrm{L}$ & \\
\hline & N6-R1 & CTT RTA RTG RAG TCC GAT GTT & $15 \mu \mathrm{L}$ & \\
\hline & N6-R2 & GAT TCC TAT YAG SAG GCT TAC & $15 \mu \mathrm{L}$ & \\
\hline & N6-R3 & GAT TCC TAT YAG SAI ICT TAC & $15 \mu \mathrm{L}$ & \\
\hline \multirow{4}{*}{ N7 assay } & N7-F1 & GTT GAA TTA ATW AGA GGA AGR CC & $20 \mu \mathrm{L}$ & \\
\hline & N7-F2 & AGA GGC YAA ATA YGT RTG GTG & $20 \mu \mathrm{L}$ & \\
\hline & N7-FAM & FAM-CCT ATG TGG RAG CCC ATT CCC AGT-BHQ1 & $3 \mu \mathrm{L}$ & \\
\hline & N7-R & GA TYT GTG CCC CAT CRG GGA & $40 \mu \mathrm{L}$ & [16] \\
\hline \multirow{7}{*}{ N8 assay } & N8-F1 & TCC ATG YTT TTG GGT TGA RAT GAT & $15 \mu \mathrm{L}$ & [16] \\
\hline & N8-F2 & CTG ATC TCT CTT ACA GGG TTG & $15 \mu \mathrm{L}$ & This study ${ }^{2}$ \\
\hline & N8-F3 & TCC ATG YTT TTG GGT IGA AAY GAT & $15 \mu \mathrm{L}$ & [16] \\
\hline & N8-FAM1 & FAM-TCH AGY AGC TCC ATT GTR ATG TGT GGA GT-BHQ1 & $6 \mu \mathrm{L}$ & [16] \\
\hline & N8-FAM2 & FAM-TGC CCA GTG ACA CTC CAA GAG GGG AA-BHQ1 & $6 \mu \mathrm{L}$ & This study ${ }^{2}$ \\
\hline & N8-R1 & GCT CCA TCR TGC CAY GAC CA & $20 \mu \mathrm{L}$ & [16] \\
\hline & N8-R2 & GTG CAT GAA CCG ACA AAT TGA G & $20 \mu \mathrm{L}$ & This study ${ }^{2}$ \\
\hline \multirow{3}{*}{ N9 assay } & N9-F & AGY ATA GTA TCR ATG TGT TCC AG & $40 \mu \mathrm{L}$ & [14] \\
\hline & N9-FAM & FAM-TTC CTR GGA CAA TGG RAC TGG CC-BHQ1 & $3 \mu \mathrm{L}$ & [16] \\
\hline & N9-R & GTA CTC TAT TYT AGC CCC RTC & $40 \mu \mathrm{L}$ & This study $^{2}$ \\
\hline \multirow{4}{*}{ NDV assay } & NDF & GAG CTA ATG AAC ATT CTT TC & $12.5 \mu \mathrm{L}$ & \multirow{4}{*}{ [23] } \\
\hline & NDR & AAT AGG CGG ACC ACA TCT G & $12.5 \mu \mathrm{L}$ & \\
\hline & ND-FAM1 & FAM-TCA TTC TTT ATA GAG GTA TCT TCA TCA TA-BHQ1 & $4 \mu \mathrm{L}$ & \\
\hline & ND-FAM2 & FAM-TCA TAC ACT ATT ATG GCG TCA TTC TT-BHQ1 & $4 \mu \mathrm{L}$ & \\
\hline
\end{tabular}


Table 2. Cont.

\begin{tabular}{|c|c|c|c|c|}
\hline Subtype & Designation & Sequence $5^{\prime} \Rightarrow 3^{\prime}$ & Amount 1 & Reference \\
\hline \multirow{7}{*}{ IBV assay } & IBV-F1 & CAG TCC CDG ATG CNT GGT A & $25 \mu \mathrm{L}$ & \multirow{7}{*}[24]{} \\
\hline & IBV-F2 & CAG TCC CDG ACG CGT GGT A & $25 \mu \mathrm{L}$ & \\
\hline & IBV-F3 & GCT TTT GAG CCT AGC GTT & $5 \mu \mathrm{L}$ & \\
\hline & IBV-FAM1 & FAM-ACT GGA ACA GGA CCD GCC GCT GAC CT-BHQ1 & $6 \mu \mathrm{L}$ & \\
\hline & IBV-FAM2 & FAM-CAC CAC CAG AAC CTG TCA CCT C-BHQ1 & $2 \mu \mathrm{L}$ & \\
\hline & IBV-R1 & CCT TWS CAG MAA CMC ACA CT & $25 \mu \mathrm{L}$ & \\
\hline & IBV-R2 & GCC ATG TTG TCA CTG TCT ATT G & $5 \mu \mathrm{L}$ & \\
\hline \multirow{3}{*}{ IC-2 } & EGFP-1-F & GAC CAC TAC CAG CAG AAC AC & $5 \mu \mathrm{L}$ & \multirow{3}{*}{ [25] } \\
\hline & EGFP-10-R & CTT GTA CAG CTC GTC CAT GC & $5 \mu \mathrm{L}$ & \\
\hline & EGFP-HEX & HEX-AGC ACC CAG TCC GCC CTG AGC A-BHQ1 & $3.75 \mu \mathrm{L}$ & \\
\hline & & \multicolumn{3}{|c|}{$\begin{array}{l}{ }^{1} \text { A stock mix of } 200 \mu \mathrm{L} \text { was produced for each assay; the amount in } \mu \mathrm{L} \text { of a } 100 \text { pmol } \mu \mathrm{L}^{-1} \text { solution of each } \\
\text { primer and probe for the stock mix is given here. } 0.1 \times \text { TE buffer was then added up to a final volume of } 200 \mu \mathrm{L} \text {. } \\
\text { Finally, } 1 \mu \mathrm{L} \text { of the stock mix was used per PCR reaction. }{ }^{2} \text { Positions shown in red have changed in comparison to } \\
\text { the RITA-1 array. Oligonucleotides shown completely in red have been newly designed in this study. IC-Internal } \\
\text { control system based on an RNA run-off transcript of a fragment of the EGFP gene [25]. }\end{array}$} \\
\hline
\end{tabular}

\subsection{RNA Extraction}

Viral RNA was extracted from infected MDCK cell cultures supernatants or allantoic fluids of embryonated chicken eggs using the NucleoMag ${ }^{\circledR}$ VET Kit (Macherey-Nagel $\mathrm{GmbH} \& \mathrm{Co}$. KG, Düren, Germany) according to the manufacturer's instructions. Clinical material (swab samples) was extracted manually using the Qiagen Viral RNA kit (Qiagen, Hilden, Germany) or by the Qiagen MagAttract Kit operated on a KingFisher Biosprint96 device (Qiagen). Samples sent on FTA cards were extracted as described by [26], using the QIAamp Viral RNA Mini Kit (Qiagen). Nucleic acids were eluted in $70 \mu \mathrm{L}$ of nuclease-free water, and aliquots of $10 \mu \mathrm{L}$ were stored at $-20^{\circ} \mathrm{C}$ until use.

\subsection{Plate Design}

The layout of the RITA-2 array aimed at economizing space by integrating several targets into duplex PCR reactions. As depicted in Figure 1a, the layout allows the testing of four samples simultaneously on one plate. Batches of plates ready-for-use were prepared and stored at $-20^{\circ} \mathrm{C}$ by pipetting $1 \mu \mathrm{L}$ of primer-probe stock mixes into the fixed positions as shown in Figure 1a before freezing.

In the same way, strips of eight or four wells were prepared with single PCR reactions decoupled from the RITA-2 design and recombined with previously published pathotyping RT-qPCRs H5-LP, H5-HP Pan, H5-HP 2.3.4.4 [17] or H7-LP and H7-HP [18] (Figure 1b).

\subsection{Set-Up of RT-qPCR Reactions}

RT-qPCRs were run on a Bio-Rad CFX 96 real-time PCR machine (Bio-Rad, Munich, Germany) in the 96-well format using the AgPath-ID One-Step kit (Applied Biosystems, Foster City, CA, USA) and non-skirted, low profile, white qPCR 96-well plates (RT-PL96OPWSA, Eurogentec, Liège, Belgium). For 8- and 4-well designs (Figure 1b) also $0.2 \mathrm{~mL}$ 8-Tube PCR Strips (low profile, white \#TLS0851, Bio-Rad, Munich, Germany) were used. A heterologous internal control system (IC-2) was used to check the performance of reverse transcription and PCR amplification [26]. Details of the reaction mix set-up are shown in Table 3, whereas $\times$ should be understood as multiplication sign. Primer-probe stock mixes had already been pipetted into plates at fixed positions, as shown in Figure 1. Fully prepared plates were sealed and kept frozen at $-20^{\circ} \mathrm{C}$ until use. Plates were prepared in batches of $20-40$ plates. The total PCR volume of a single reaction comprised $12.5 \mu \mathrm{L}$ to which $2.5 \mu \mathrm{L}$ of extracted RNA was added. CFX96 machines were programmed as follows: Reverse transcription $45^{\circ} \mathrm{C}$ for $10 \mathrm{~min}$, initial denaturation $95^{\circ} \mathrm{C}$ for $10 \mathrm{~min}, 45$ cycles of denaturation $95^{\circ} \mathrm{C}$ for $15 \mathrm{~s}$, annealing/reading (of FAM and HEX channels) $56.5^{\circ} \mathrm{C}$ for $20 \mathrm{~s}$, and elongation $72{ }^{\circ} \mathrm{C}$ for $30 \mathrm{~s}$. The $\mathrm{Cq}$ threshold was set to $<40$. 


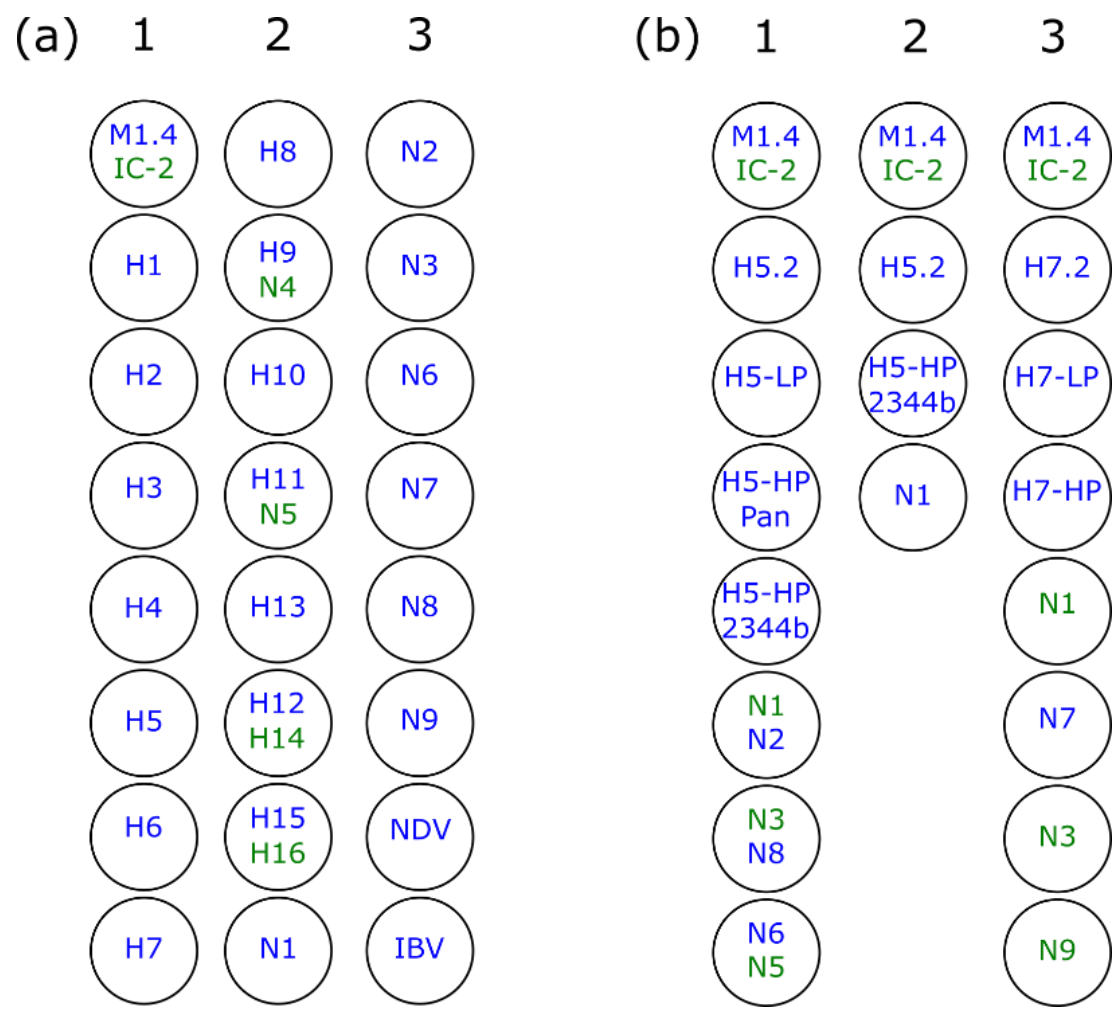

Figure 1. (a) 24-well plate layout (1-3) of the RITA-2 array allowing simultaneous testing of four clinical samples on a whole 96-well plate. Some reactions were decoupled from the RITA-2 format and newly recombined with additional reactions in eight- and four-well format for use in routine diagnostics tailored for epizootic outbreaks of notifiable AIV (b) 1- Eight-well layout for sub- and pathotyping of Eurasian H5 viruses, 2- Four-well layout for sub- and pathotyping of viruses encountered during the current (autumn 2021) HPAIV H5N1 epizootic, 3- Eight well layout for sub- and pathotyping of Eurasian H7 viruses. Subtype color indicates the type of reporter dye, blue-FAM, green-HEX.

Table 3. Reaction volumes used for individual and arrayed RT-qPCRs.

\begin{tabular}{cccc}
\hline \multirow{2}{*}{$\begin{array}{c}\text { RNA/Mastermix AgPath-ID } \\
\text { One-Step RT-PCR }\end{array}$} & Single Reaction & $\begin{array}{c}\text { 24 Reactions } \\
\text { (1 Sample) }\end{array}$ & $\begin{array}{c}\text { 96 Reactions } \\
\text { (4 Samples) }\end{array}$ \\
\cline { 2 - 4 } & $\mathbf{1} \times$ & $\mathbf{2 6 \times}$ & $\mathbf{1 0 0 \times}$ \\
\hline RNase free water & $2.25 \mu \mathrm{L}$ & $58.5 \mu \mathrm{L}$ & $225 \mu \mathrm{L}$ \\
\hline $2 \times$ RT-PCR Buffer & $6.25 \mu \mathrm{L}$ & $162.5 \mu \mathrm{L}$ & $625 \mu \mathrm{L}$ \\
\hline RT-PCR Enzyme Mix & $0.5 \mu \mathrm{L}$ & $13 \mu \mathrm{L}$ & $50 \mu \mathrm{L}$ \\
\hline Primer-Probe mix ${ }^{\text {Thy }}$ & $1 \mu \mathrm{L}$ & $26 \mu \mathrm{L}$ & $100 \mu \mathrm{L}$ \\
\hline Sample RNA & $2.5 \mu \mathrm{L}$ & $65 \mu \mathrm{L}$ & $2.5 \mu \mathrm{L} / \mathrm{well}$ \\
\hline Total volume & $\mathbf{1 2 . 5} \mu \mathrm{L}$ & $\mathbf{2 9 9} \boldsymbol{\mu L}$ & $\mathbf{1 0 0 0} \mu \mathrm{L}$ \\
\hline Template & $2.5 \mu \mathrm{L} / \mathrm{well}$ & $2.5 \mu \mathrm{L} / \mathrm{well}$ & $2.5 \mu \mathrm{L} / \mathrm{well}$ \\
\hline
\end{tabular}

${ }^{1}$ Primer-probe mixes had already been pipetted into plates at fixed positions as shown in Figure 1.

\subsection{Preparation of Positive Controls}

Different plate batches were evaluated by a set of four positive controls, each consisting of RNA of five different viruses, as shown in Table 4 . RNA of individual viruses was mixed in RNA safe buffer [16] $\left(0.05 \% v / v\right.$ Tween $20,0.05 \% w / v$ sodium azide, $50 \mathrm{ng} \mu \mathrm{L}^{-1}$ of carrier RNA [poly(A) homopolymer; Amersham Biosciences, Piscataway, NJ, USA]) to ensure a $\mathrm{Cq}$ value of $28-32$ in the M-PCR. PTCs were frozen at $-80{ }^{\circ} \mathrm{C}$ until use. The 
identification of strains used to compile the PTCs is given in Supplementary Materials

Table S2. Each batch of plates was also checked with a no-template control.

Table 4. Identity of virus isolates used as positive controls for RITA-2 batch evaluations.

\begin{tabular}{|c|c|c|c|}
\hline Positive Control & Subtype & Strain & Cq Value/Reaction \\
\hline \multirow{5}{*}{ PTC-1 } & H1N1 & A/Mallard/Germany/R193/09 & $23-25$ \\
\hline & H5N6 & A/White stork/Germany/AR251/2018 & $21-23$ \\
\hline & H9N2 & A/Chicken/Egypt/AR538/2017 & $22-25$ \\
\hline & H13N8 & A/Larus ridibundus/Germany/R2064/2006 & $24-26$ \\
\hline & IBV-1 & AI20298/2019 & $23-25$ \\
\hline \multirow{5}{*}{ PTC-2 } & $\mathrm{H} 2 \mathrm{~N} 3$ & A/Mallard/Germany/Wv677/04 & $23.65-25$ \\
\hline & H6N2 & A/Turkey/Mass/3740/65 & $22-24$ \\
\hline & H10N7 & A/Mallard/Germany/1490/09 & $22-24$ \\
\hline & H14N5 & A/Mallard/Gurjev/263/82 & $26-27$ \\
\hline & IBV & AI20298/2019 & $23-25$ \\
\hline \multirow{5}{*}{ PTC-3 } & H3N8 & A/Mallard/Germany/R1648/07 & $23-25$ \\
\hline & H7N7 & A/Greylag goose/Germany/AR942/2015 & $22-24$ \\
\hline & H11N9 & A/Mallard/Föhr/Wv1499-1503/03 & $22-24$ \\
\hline & H15N9 & A/Shearwater/West Australia/2576/79 & $22-24$ \\
\hline & NDV-1 & ND/Lentogenic/713/2016 & $22-24$ \\
\hline \multirow{5}{*}{ PTC-4 } & H4N6 & A/Mallard/Germany/R485/3/08 & $21-23$ \\
\hline & $\mathrm{H} 8 \mathrm{~N} 4$ & A/Anas latyrhynchos/Germany/R2167/2009 & $22-24$ \\
\hline & H12N5 & A/Duck/Alberta/60/76 & $21-23$ \\
\hline & H16N3 & A/Herring gull/Germany/R2788/06 & $23-25$ \\
\hline & NDV-2 & ND/Velogenic & $22-24$ \\
\hline
\end{tabular}

\subsection{Statistical Analyses}

Statistical analyses for sensitivity, specificity, intra-, and inter-assay variation were performed using the SigmaPlot software, version 11 (Systat Software Inc., Duesseldorf, Germany). Spearman's rank correlation and Student's t-test were employed. $p<0.05$ was considered significant.

\section{Results}

\subsection{Evaluation and Selection of Oligonucleotide Sets}

In silico analyses of comprehensive sequence alignments for each HA and NA subtype of Eurasian origin revealed, for the majority of subtypes, a within-subtype variation that was too wide to be covered by a single set of primers and probe; inclusion of sequences of American or Australian origin grossly increased such variation. Therefore, it was decided, as a continuation of the array strategy of RITA-1, to restrict oligonucleotide selection to Eurasian viruses. In order to avoid a large number of degenerate positions, two (e.g., H1, $\mathrm{H} 5, \mathrm{~N} 8$ ) and even three separate sets (H3) of primers and probes had to be designed to cover the full width of sequence variation of these subtypes (Table 2). The PCRs were du- or triplexed within each subtype and probes specific for the same subtype labeled with the same reporter dye. As indicated by the red color of nucleotides in Table 2, the majority of oligonucleotides used in RITA- 1 had to be modified or fully replaced to qualify for inclusion into RITA-2. Primer and probe sets were tested extensively against selected reference isolates of the matching and closely related subtypes with several rounds of 
optimization. The final selection was then successfully tested against all homosubtypic isolates as listed in Table 1.

\subsection{Extended Target Spectrum of RITA-2}

To economize space on the final PCR array, RT-qPCRs specific for several different subtypes were duplexed using FAM and HEX reporter dyes (Figure 1a: M/IC2; H9/N4; H11/N5; H12/H14; H15/H16); care was taken to combine subtypes that have not been detected so far in nature to avoid competition during amplification.

While RITA-1 provided no reactions to identify subtypes H14 and H15, these have now been added to RITA-2. Figure 2 shows that these assays have a sensitivity comparable to the generic M-PCR. In addition, the H15 assay did not cross-react to the closely related subtypes $\mathrm{H} 7$ and H10. Unfortunately, only a single reference isolate was available each for AIV subtypes H14 and H15.

(a)
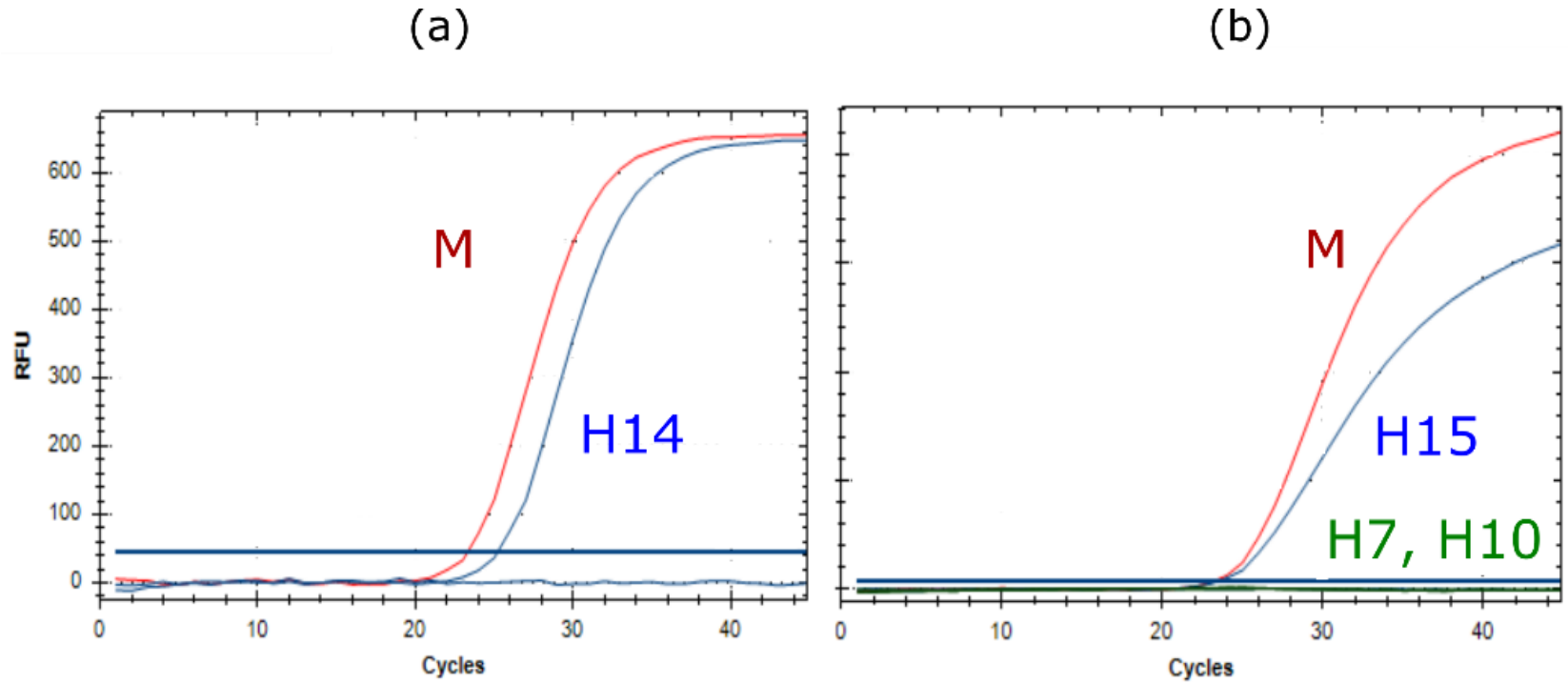

Figure 2. RT-qPCRs specific for avian influenza virus subtypes (a) H14 (A/Mallard/Gurjev/263/82), and (b) H15 (A/Shearwater/West Australia/2576/79), in comparison to influenza A virus-generic M-PCR (red, in (a,b)). Subtypes H7 and H10 (green in (b)), closely related to H15 did not cross-react.

According to recent data from Egypt, poultry flocks showing respiratory disease and increased mortality were often found to suffer from co-infections of AIV with NDV and/or IBV $[23,24]$. Likewise, viral isolates were found to harbor a mix of AIV, NDV, and/or IBV [27-29]. In addition, NDV and IBV infections are among the most important differential diagnoses of HPAI in poultry. Therefore, in RITA-2, NDV- and IBV-specific RT-qPCRs have been included. Rather than designing new assays, approved published methods have been adopted here (Table 2).

Routine use of RITA- 1 for subtyping clinical samples isolates revealed a lower sensitivity of several subtype-specific assays compared to the generic M-PCR. Examples are shown in Figure 3. Re-designing primers and/or probes or selecting completely new target regions re-established sensitivity of the RITA-2 assays to the level of the M-PCR.

\subsection{Analytical Sensitivity}

All subtype-specific assays were evaluated with all the available matched subtypes and compared to the generic M-PCR. A wide range of isolates regarding time, place of origin, and host species was used. The analyses showed that most of the subtype-specific assays attained the level of sensitivity of the M-PCR as indicated by correlation coefficients $>0.93$ (Figure 4). For some assays (H2, H6, H11, N2, N7), however, a statistically significant $(p<0.05)$ lower sensitivity of up to $3 \mathrm{Cq}$ value on average was calculated. For H1, H3, and N1 subtypes, the host species origin was found to modulate sensitivity (Figure 5), 
and targets of non-avian origin were detected with significantly lower sensitivity due to mismatches in primers and/or probes. The $\mathrm{H} 1$ subtypes of human and swine-origin were particularly negatively affected. In order to address these findings in routine diagnostic settings, clinical samples with a viral load of $\mathrm{Cq}>35$ (as measured by a generic influenza A virus RT-qPCR) were not assigned to examination in the RITA-2 array.

(a)

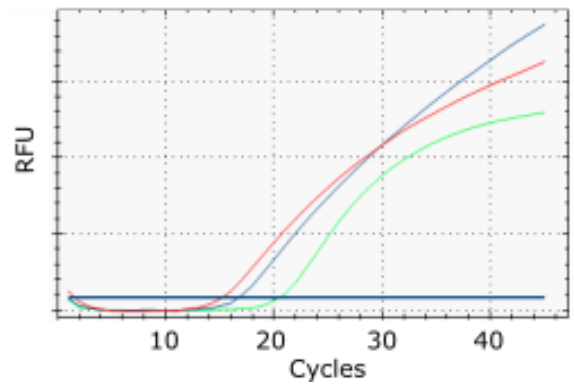

(b)

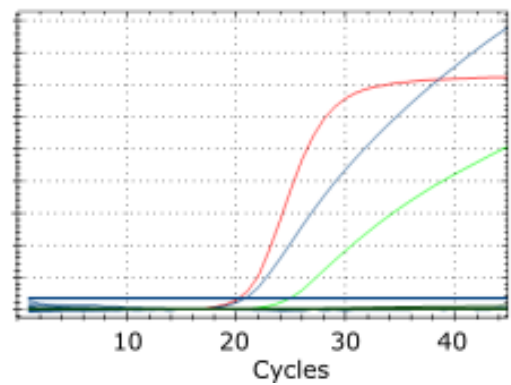

(c)



Figure 3. Improved sensitivity of RT-qPCRs specific for avian influenza virus subtypes (a) H3 (A/Mallard/Germany/R1648/07 [H3N8]), (b) N2 (A/Chicken/Egypt/AR538/2017 [H5N2 hp]), and (c) N4 (A/Mallard /Germany/R2167/2009 [H8N4]) in RITA-2 (blue) compared to RITA-1 (green). Generic M-specific amplification curves are shown in red.
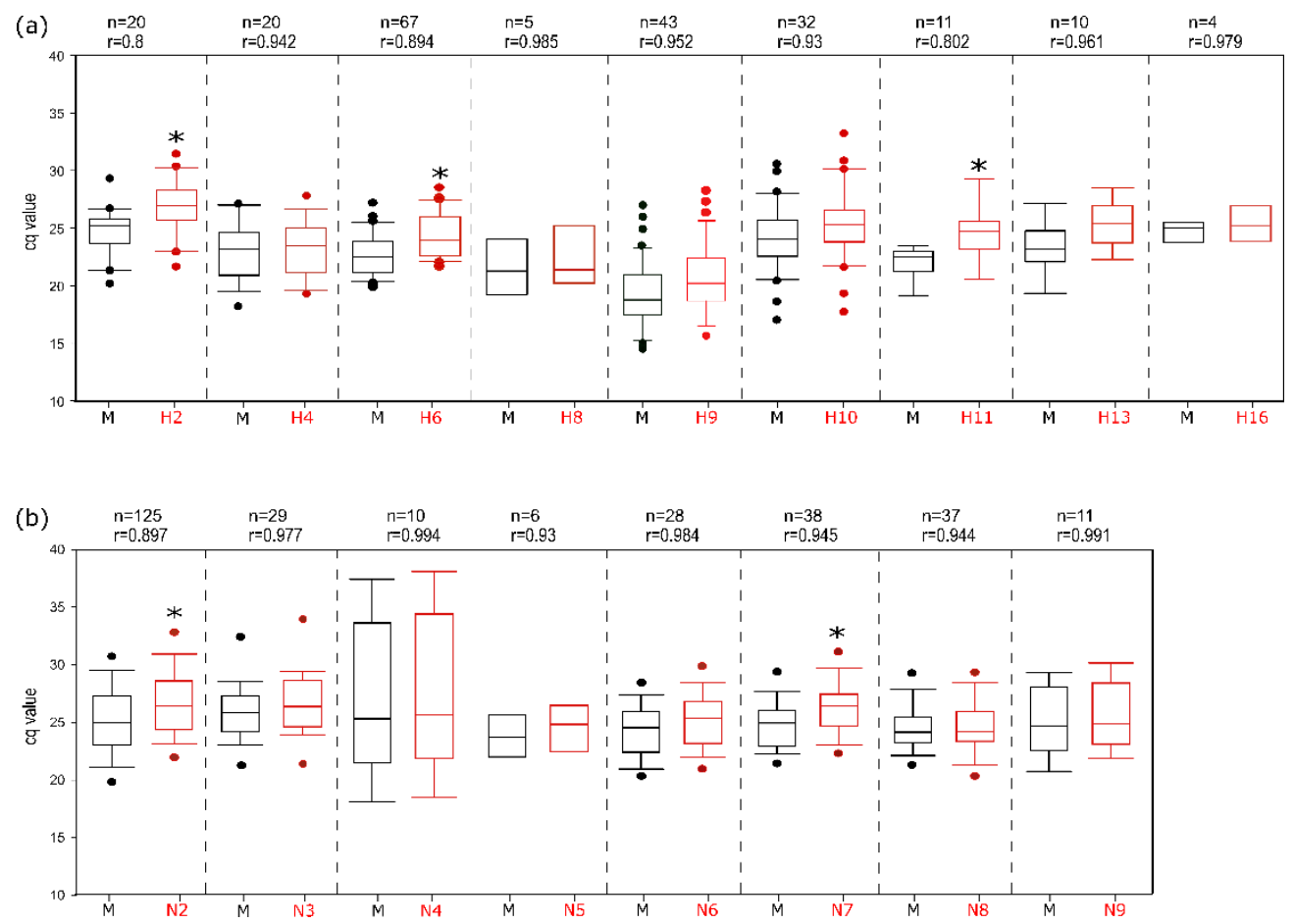

Figure 4. Subtype-specific analytical sensitivity of the RITA-2 array compared to the generic MRTqPCR assay. (a) HA subtypes 2, 4, 6, 8, 9, 10, 11, 13, and 16; for subtypes H14 and H15, only a single isolate was available (s. Figure 2). (b) NA subtypes 2-9. n-Number of isolates tested, r-Spearman's rank correlation coefficient, ${ }^{*}$ - statistically significant difference between the $\mathrm{Cq}$ values of the generic and the subtype-specific RT-qPCRs. Dots define outliers.

Notifiable AIV of subtypes H5 and H7 received specific dedication (Figure 6): RITA2 assays detected RNA of these subtypes with high sensitivity, independently of the pathotype and the clade of $\mathrm{H} 5$ viruses of the goose/Guangdong lineage. 


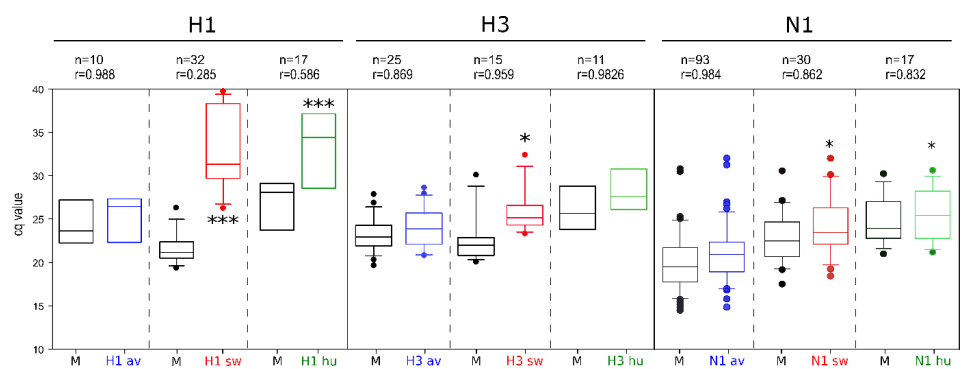

Figure 5. Influence of host origin of virus isolates on the analytical sensitivity of the RITA-2 array. $n$-Number of isolates tested, $\mathrm{r}$-Spearman's rank correlation coefficient, ${ }^{*}$ - Statistically significant difference. ***-Highly significant difference; av, sw, hu—avian (blue), swine (red), human (green) host origin. Dots define outliers.
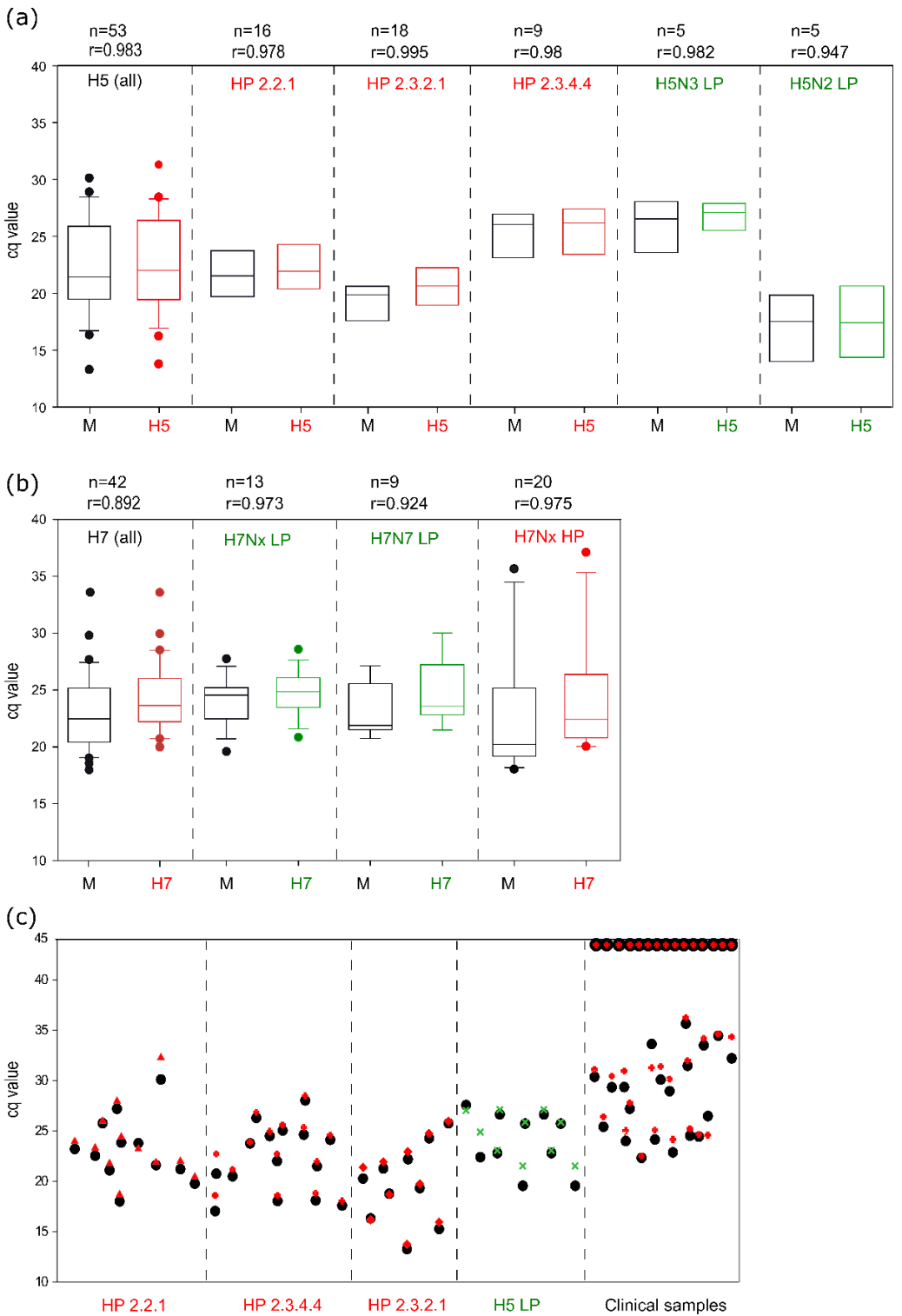

Figure 6. Analytical sensitivity of the RITA-2 array for notifiable avian influenza viruses of HA subtypes $\mathrm{H} 5$ (a) and $\mathrm{H} 7$ (b), stratified by phylogenetic lineage and pathotype (HP-red, LP—green) and (c) pairwise comparison of $\mathrm{Cq}$ values for individual isolates or clinical samples (including H5negative ones) obtained by generic M-PCR (black dot) and the H5 subtype-specific RITA-2 assay (colored symbols). $n$-number of tested isolates, $\mathrm{r}$-Spearman's rank correlation coefficient. 


\subsection{Analytical Specificity}

In RITA-1, several assays revealed minor intersubtypic cross-reactions, particularly between subtypes $\mathrm{H} 1 / \mathrm{H} 6, \mathrm{H} 2 / \mathrm{H} 5$, and $\mathrm{H} 7 / \mathrm{H} 10 / \mathrm{H} 15$ which are known to be genetically closely related. Therefore, special care was taken to re-design primers and probes to avoid such cross-reactions. Based on published phylogenetic panoramas for the HA and NA subtypes [30,31], a selected pattern of closely related subtypes was used to validate analytical specificity.

As shown in Figure 7, upper left panel, RITA-1 produced a highly specific signal for $\mathrm{H} 2$ when tested with an $\mathrm{H} 2$ isolate but also an $\mathrm{H} 5$ virus gave a (significantly weaker) positive signal in the $\mathrm{H} 2$ assay; the same was seen vice versa for the $\mathrm{H} 5$ assay. The problem was even more complex for $\mathrm{H} 7$ viruses, which are closely related to subtypes $\mathrm{H} 10$ and H15: In RITA-1, an H7 virus gave a highly specific signal in the H7 assay, but also H10 and H15 viruses tested (false-) positive in the H7 assay (Figure 7, lower left panel). Similarly, an $\mathrm{H} 10$ virus produced a weakly positive specific signal in the H7 assay. In RITA-2, these cross-reactions no longer exist (Figure 7, right panels). Similar results were obtained for all assays tested against the pattern of closely related subtypes. Thus, the RITA-2 array has increased in specificity.

RITA-1



$\mathrm{H} 7$

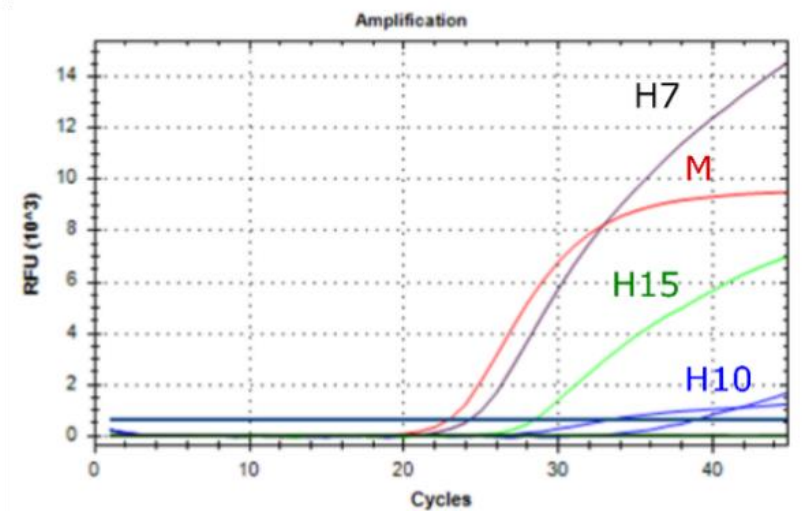

RITA-2
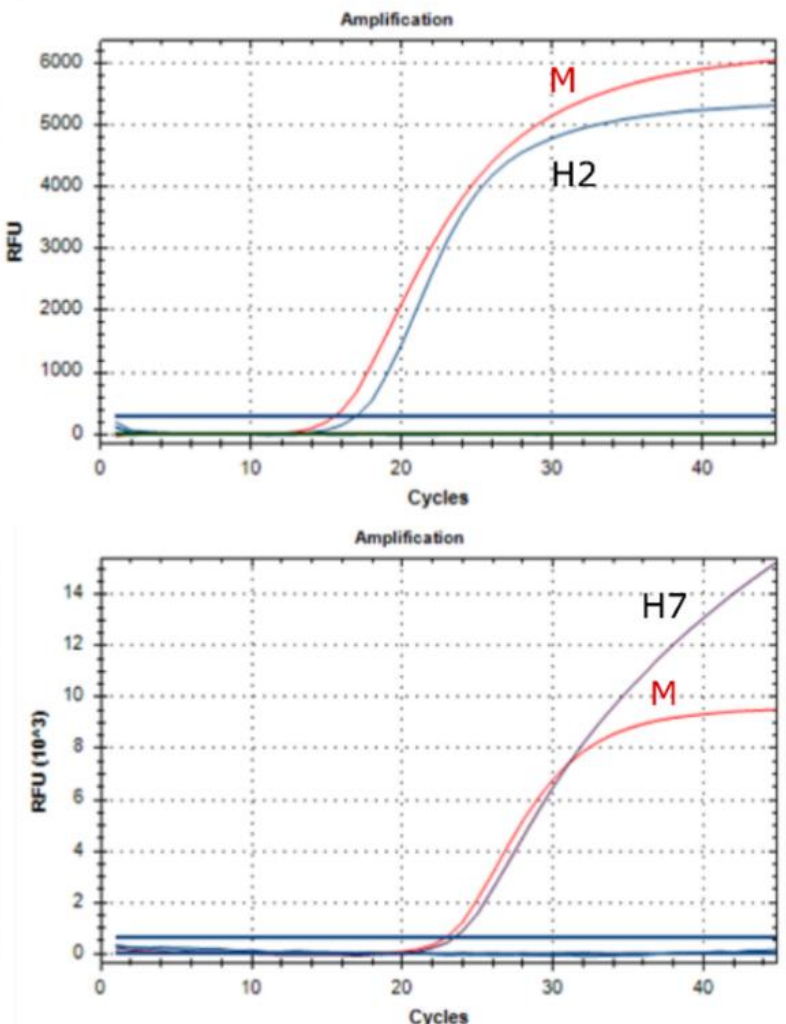

Figure 7. Intersubtypic cross-reactions of RITA-1 are resolved in re-designed RITA-2 assays. Here the following strains were used: H2 (A/Mallard/Germany/Wv677/04 [H2N3]), H5 (A/White stork/Germany/AR251/2018 [H5N6 hp]), H7 (A/Greylag goose/Germany/AR942/2015 [H7N7]), H10 (A/Mallard/Germany/1490/09 [H10N7]) and H15 (A/Shearwater/West Australia/2576/79 [H15N9]).

\subsection{Assay Robustness}

Duplicate runs of selected reference samples for all $16 \mathrm{HA}$ and nine NA assays plus IBV and NDV targets on the same plate were used to evaluate intraassay variation. Interassay variation was investigated using two different thermal cyclers and two different 
plates for the same selected samples on two different days. As shown in Tables S3 and S4, the standard deviations and covariances calculated suggest excellent assay robustness.

\subsection{Performance Characteristics with Clinical Samples}

The diagnostic performance of RITA-2 was evaluated with a set of 60 clinical samples from poultry originating from Egypt and Bangladesh. RITA-2 succeeded in differentiating subtypes and detecting IBV and/or NDV with high sensitivity and specificity, as shown in Table 5. In RITA-2, all subtypes specified for these samples by other RT-PCRs and/or sequence analysis were confirmed (H5, H9, N1, N8, and N2). Some of these samples were also tested by RITA-1 but failed to detect the H9 subtype at lower viral loads. RITA- 2 takes advantage of a set of primers and probes that we updated previously [23], which enabled detection of recent $\mathrm{H} 9 \mathrm{~N} 2$ viruses of the G1 lineage circulating in northern Africa with much higher sensitivity than an older $\mathrm{H} 9$ protocol [32]. Also, mixed infections of different AIV subtypes were detected; these results extended to co-infections with IBV and NDV in two samples, as depicted in Figure 8.

Table 5. Comparison of the results of clinical samples obtained with RITA-2 and by sequencing or subtyping with other RT-PCRs. Results for sample originating from Germany are shown in Table S2.

\begin{tabular}{cccccc}
\hline Country & Species & $\begin{array}{c}\text { No. of } \\
\text { Farms }\end{array}$ & RITA-2 & $\begin{array}{c}\text { Subtyping/ } \\
\text { Other PCRs }\end{array}$ & Sequencing \\
\hline Egypt & Chicken & 2 & H5, N8 & H5, N8 & H5 HP, N8 [22] \\
\hline Egypt & Turkey & 2 & H5, N8 & H5, N8 & H5 HP, N8 [22] \\
\hline Egypt & Ducks & 1 & H5, N8 & H5, N8 & H5 HP, N8 \\
\hline Egypt & Ducks & 7 & H5, N8 & H5, N8 & H5 HP \\
\hline Egypt & Chicken & 3 & H9, N2 & H9, N2 & H5, N22] \\
\hline Egypt & Chicken & 2 & H5, N1 & H5, N1 & H5, N8 HP \\
\hline Egypt & Duck & 5 & H5, N8 & H5, H9, N2 & H5 HP [22] \\
\hline Egypt & Chicken & 5 & H5, H9, N8, N2 & H5, N2 & H5 HP, N2 [22] \\
\hline Egypt & Chicken & 3 & H5, N2 & H5, H9, N8, N2 & H5, H9, N8, N2 [10] \\
\hline Egypt & Chicken & 2 & H5, H9, N8, N2 & H5, H9, N8, N2, IBV, NDV & H5, H9, N8, N2, IBV [10] \\
\hline Egypt & Chicken & 1 & H5, H9, N8, N2, IBV, NDV & H5, N8, IBV, NDV & H5, N8, IBV [10] \\
\hline Egypt & Chicken & 1 & H5, N8, IBV, NDV & H4, N6 & H4, N6 [23] \\
\hline Bangladesh & Duck & 13 & H4, N6 & &
\end{tabular}

In addition, we decoupled single assays from the RITA-2 array and re-arranged smaller arrays of eight or four wells (Figure 1b) comprising RT-qPCRs for: (i) generic AIV detection, (ii) $\mathrm{H} 5$ or H7 sub- and pathotyping, and (iii) fitting NA subtyping. This step was taken to guarantee high throughput of a demanding daily sample size during HPAI epizootics experienced in Europe 2016/17 and 2020/21. We analyzed a total of 63 clinical samples from the recent 2020/21 HPAI H5 epizootic in Europe (Supplementary Materials Table S3) and show that careful selection of single assays decoupled from RITA-2 allowed a full diagnosis regarding $\mathrm{H} 5$ sub- and pathotype as well as (in most cases) the NA subtype with a grossly reduced turn-around time as compared to the full RITA-2 or single RT-qPCRs and nucleotide sequencing for pathotyping. 
(a)

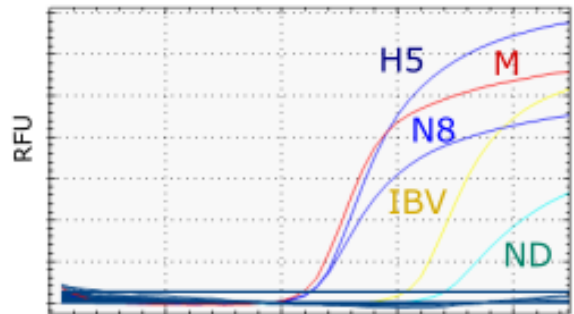

(c)

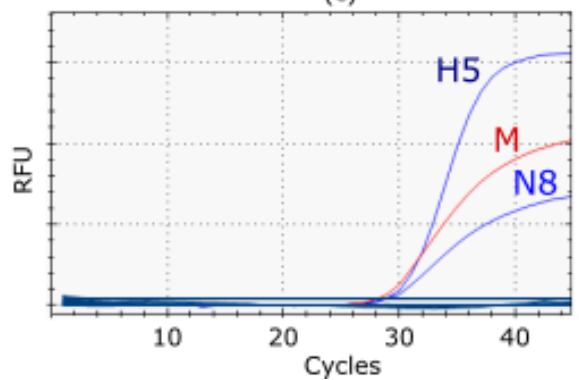

(b)

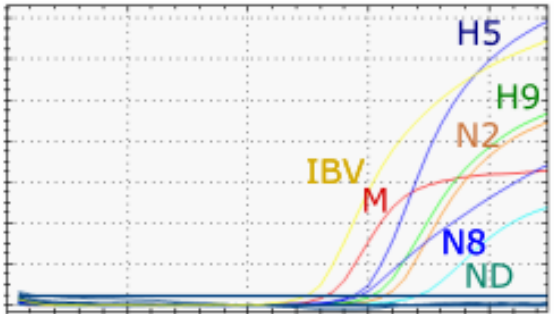

(d)



Figure 8. Examples of RITA-2 analysis of clinical (cloacal swab) samples from poultry flocks in Egypt detecting mixed infections of several AIV subtypes (a) Mixed infection with AIV H5N8, IBV and NDV; (b) Mixed infection with AIV H5, H9, N2, N8 IBV and NDV; (c) Mono-infection with AIV H5N8; (d) Mono-infection with AIV H5N2.

\section{Discussion}

Diagnostic tools for avian influenza viruses (AIV) are constantly challenged due to reassortment and genetic drift of these viruses. Recently, RT-qPCRs have been established as standards for rapid and sensitive diagnosis [4]. Due to the rapid evolutionary diversification of the AIV sequence cloud, particularly affecting the HA segment, subtyping RT-qPCRs are under exceptionally high pressure to adapt to the fluctuations of such clouds. Ideally, assays should be inclusive for all sequences published for a single subtype and, at the same time, exclusive for all sequences not clustering with this subtype. Suitably conserved but subtype-specific target sequences in the HA and NA genome segments become limited in reciprocal relation to the growing number of sequences in databases, as our in-silico study of comprehensive alignment sets has revealed.

To re-establish high sensitivity and specificity of arrayed influenza virus subtypespecific RT-qPCRs for recently circulating viruses, we re-designed primers and probes previously published by Hoffmann et al. [16] and re-assembled those PCRs into an economized PCR array, termed RITA-2. An acceptable balance between a highly sensitive broad reactivity and full subtype specificity was finally achieved using multiplexed Taqman ${ }^{\circledR}$ based technology. The use of two fluorescent marker dyes allowed integration of PCRs and reduced the set of previously 32 subtyping PCRs of RITA- 1 to 24 in RITA-2. In addition, two further AIV subtypes (H14, H15) and targets for NDV and IBV, two important differential diagnoses of AIV infections in poultry, were accommodated into the array. The assay provides considerable versatility and robustness as all 24 wells for a single sample can be pipetted using a multichannel pipette and a single master mix per sample. The plates can be prepared in advance in batches with primers and probes pipetted into the correct positions and stored at $-20^{\circ} \mathrm{C}$. A storage time of nine months in our hands did not lead to a loss of sensitivity. Cutting the plates if the full range of four samples per plate is not required can be accomplished as well. Reducing the total volume of the RT-qPCR reactions from $25 \mu \mathrm{L}$ (RITA-1) to $12.5 \mu \mathrm{L}$ per reaction further adds to cost reduction in RITA-2.

A continuing emergence of AIV variants characterized the evolution of highly pathogenic (HP) AIV of the Chinese Gs/GD lineage over the last two decades. NA subtype switching and an accelerated diversification of HA sequences leading to an intricate fragmentation into various phylogenetic clades, subclades, and lineages have been a hallmark of these 
viruses [33]. Appropriate diagnostic tools should be able to detect such diverse viruses, and the $\mathrm{H} 5$ assay implemented in RITA-2 achieves this goal as demonstrated by detecting representative HPAI H5 viruses from at least four recent clades (Figure 6a). Severe epizootic outbreaks of Gs/GD HP H5 viruses have been witnessed in Europe during the winter seasons of 2016/17 (mainly H5N8), 2020/21 (mainly H5N8, but also N1, N3, N4, and N5), and 2021/22 (mainly H5N1) [34,35]. Single subtyping assays of RITA-2 and pathotyping RT-qPCR published by Naguib et al. and Graaf et al. $[17,18]$ were customized and rearranged into 8- and 4-well arrays (Figure $1 \mathrm{~b}$, Table S2). In cases such as the mentioned HPAI epizootics, when most AIV-positive samples are expected to be dominated by a certain sub- and pathotype, smaller arrays can be used to save time and costs to establish a final diagnosis (Table S2).

Mixed infections with different influenza A virus subtypes are a prerequisite of reassortment. In addition, co-infections of AIV and other viral avian pathogens with clinical impact have recently been found at increasing frequency in poultry in countries like Egypt [23,24,27-29] and Bangladesh [36]. In Egypt, such co-infection gave rise to a diffuse clinical picture, termed "respiratory disease complex" in gallinaceous poultry [27]. We show here that RITA-2 detects mixed infections in clinical samples even if these targets are present in grossly different concentrations (Figure 8). Separating the amplification reaction into distinct wells obviates competition effects which would decrease the sensitivity of detecting minor targets. To detect mixed infections, it is essential to understand better the viral infection dynamics in individual birds and populations. Reassortment events "in statu nascendi" can be followed using RITA-2, and has led previously to the detection of a new HPAIV subtype, H5N2, in Egypt [11]. Sensitive virus isolation techniques would essentially cover at least the same range of viruses (AIV, NDV, IBV), but often certain viruses outcompete others in such mixtures [28,29]. This would lead to a skewed impression of the actual co-infections. Next-generation sequencing focusing on a true metagenomics approach would be adequate to uncover the full range of co-infecting pathogens in a sample [34]. However, such technology is still costly and less suitable than RT-qPCR for routine diagnostic laboratories. The capabilities of RITA-2 to detect mixed infection might also be useful when analyzing the purity of virus strains based on clinical isolates that are used as diagnostic antigens in serological assays or for (autologous) vaccine production.

Emerging new subtypes and drift variants of influenza A viruses pose an ultimate challenge to their diagnostic detection by sequence-based techniques such as RT-qPCR. There is extensive natural sequence variation between AIV subtypes circulating in wild birds and poultry in the Americas and Oceania compared to Eurasia [30,31]. In silico analyses of the available sequence information did not allow the selection of a global "one-fits-all" type of RT-qPCR for all the different subtypes. Therefore, like RITA-1, the new version-2 remains restricted to the analysis of viruses and samples obtained from wild birds and poultry of Eurasia and Africa. Nevertheless, it should be possible to design a similar array for the corresponding American or Oceanian lineages. Sequence variation was also related to the host origin of the viruses and negatively affected the sensitivity of detection. In particular, the $\mathrm{H} 1$ assay was not sensitive enough to ensure proper detection of $\mathrm{H} 1$ viruses in clinical samples of swine and human origin. Other, more sensitive assays have been published that should be used for those species instead [37]. Unlike H1 viruses, $\mathrm{H} 3$ viruses were detected more efficiently also from swine and humans; this is likely due to the use of three multiplexed RT-qPCRs targeting a larger spectrum of $\mathrm{H} 3$ sequences. The re-designed N1 assay was able to pick up the N1 subtype from the swine and human origin samples.

\section{Conclusions}

RITA-2 constitutes an important technical update and improved development compared to RITA-1. It also provides functional progress through the combination of RT-qPCR for differential diagnoses of AIV infections; this serves needs to disentangle complex mixtures of viral co-pathogens (NDV, IBV) synergistically acting in respiratory disease of 
poultry. It is clear that the assay compositions arrayed here will remain subjects to change. AIV in general and Gs/GD viruses, in particular, remain a highly mobile and moving target for sequence-based diagnostic tools. Continuous reference to updated sequence databases and appropriate adaptation of primers and probes are inevitable permanent tasks. An additional functional step forward compared to RITA-1 is provided by the combination of selected RT-qPCR assays with pathotyping RT-qPCR so as to serve the needs of rapid diagnosis of HA and NA subtypes and of the pathotype in HPAI epidemics with a temporal and/or geographic restriction.

Supplementary Materials: The following supporting information can be downloaded at: https: / / www.mdpi.com/article/10.3390/v14020415/s1, Table S1. Identity of Infectious Bronchitis and Newcastle Disease virus isolates used to validate specific RT-qPCRs; Table S2. Analysis of clinical samples obtained during active or passive monitoring of wild birds and poultry in Germany, 2020-21. Table S3. Summary statistics of intra- and interassay variations of the RITA-2 array; Table S4. Raw data of inter- and intra-assay variations.

Author Contributions: Conceptualization, T.H. and M.B.; methodology, K.E.H. and A.K.A.; software, A.A.; validation, K.E.H. and A.K.A.; formal analysis, K.E.H. and A.K.A.; investigation, K.E.H., A.A. and A.K.A.; resources, T.C.M. and M.B.; data curation, T.H.; writing-original draft preparation, K.E.H., A.K.A. and T.H.; writing-review and editing, T.H. and M.F.E.-K.; visualization, K.E.H.; supervision, T.C.M., M.B., H.M.H. and M.F.E.-K.; project administration, T.H.; funding acquisition, M.F.E.-K., K.E.H. and T.C.M. (for A.K.A.). All authors have read and agreed to the published version of the manuscript.

Funding: This research was funded by a grant to K.E.H. from the Egyptian Ministry of Higher Education (Mission sector) and by a grant to A.K.A. from the Federal German Environment Agency (Umweltbundesamt), grant number FKZ 3718622360.

Institutional Review Board Statement: No segments of the study required ethical approval. Clinical samples were not collected specifically for this study but were obtained from routine diagnostic submissions.

Data Availability Statement: All data pertinent to this study are presented in tables and figures in the main text or in the Supplementary Materials.

Acknowledgments: We gratefully acknowledge the technical support provided by Aline Maksimov and Diana Parlow, Friedrich-Loeffler-Institute, and the willingness of several veterinarians in Bangladesh, Egypt, and Germany to submit clinical sample material.

Conflicts of Interest: The authors declare no conflict of interest.

\section{References}

1. Webster, R.; Govorkova, E. Continuing challenges in influenza. Ann. N. Y. Acad. Sci. 2014, 1323, 115-139. [CrossRef]

2. Ma, W.; Gramer, M.; Rossow, K.; Yoon, K.-J. Isolation and genetic characterization of new reassortant H3N1 swine influenza virus from pigs in the midwestern United States. J. Virol. 2006, 80, 5092-5096. [CrossRef]

3. Fouchier, R.A.M.; Munster, V.; Wallensten, A.; Bestebroer, T.M.; Herfst, S.; Smith, D.; Rimmelzwaan, G.F.; Olsen, B.; Osterhaus, A.D.M.E. Characterization of a novel influenza A virus hemagglutinin subtype (H16) obtained from black-headed gulls. J. Virol. 2005, 79, 2814-2822. [CrossRef]

4. Spackman, E.A. Brief Introduction to Avian Influenza Virus. Methods Mol. Biol. 2020, 2123, 83-92.

5. Horimoto, T.; Kawaoka, Y. Influenza: Lessons from past pandemics, warnings from current incidents. Nat. Rev. Microbiol. 2005, 3, 591-600. [CrossRef]

6. Poovorawan, Y.; Pyungporn, S.; Prachayangprecha, S.; Makkoch, J. Global alert to avian influenza virus infection: From H5N1 to H7N9. Pathog. Glob. Health 2013, 107, 217-223. [CrossRef]

7. Machalaba, C.C.; Elwood, S.E.; Forcella, S.; Smith, K.M.; Hamilton, K.; Jebara, K.B.; Swayne, D.E.; Webby, R.J.; Mumford, E.; Mazet, J.A.K.; et al. Global avian influenza surveillance in wild birds: A strategy to capture viral diversity. Emerg. Infect. Dis. 2015, 21, e1-e7. [CrossRef]

8. Chan, K.H.; Lam, S.Y.; Puthavathana, P.; Nguyen, T.D.; Long, H.T.; Pang, C.M.; Chan, K.M.; Cheung, C.Y.; Set, W.H.; Peiris, J.S. Comparative analytical sensitivities of six rapid influenza A antigen detection test kits for detection of influenza A subtypes H1N1, H3N2 and H5N1. J. Clin. Virol. 2007, 38, 169-171. [CrossRef]

9. Marché, S.; van den Berg, T. Evaluation of rapid antigen detection kits for the diagnosis of highly pathogenic avian influenza H5N1 infection. Avian Dis. 2010, 54, 650-654. [CrossRef] 
10. Chua, T.H.; Ellis, T.M.; Wong, C.W.; Guan, Y.; Ge, S.X.; Peng, G.; Lamichhane, C.; Maliadis, C.; Tan, S.W.; Selleck, P.; et al. Performance evaluation of five detection tests for avian influenza antigen with various avian samples. Avian Dis. 2007, 51, 96-105. [CrossRef]

11. Hassan, K.E.; King, J.; El-Kady, M.; Afifi, M.; Abozeid, H.H.; Pohlmann, A.; Beer, M.T.H. Novel Reassortant Highly Pathogenic Avian Influenza A(H5N2) Virus in Broiler Chickens, Egypt. Emerg. Infect. Dis. 2020, 26, 129-133. [CrossRef]

12. Brown, I. Advances in molecular diagnostics for avian influenza. Dev. Biol. 2006, 124, 93-97.

13. Suarez, D.L.; Das, A.; Ellis, E. Review of rapid molecular diagnostic tools for avian influenza virus. Avian Dis. 2007, 51, 201-208. [CrossRef]

14. Kalthoff, D.; Bogs, J.; Harder, T.; Grund, C.; Pohlmann, A.; Beer, M.; Hoffmann, B. Nucleic acid-based detection of influenza A virus subtypes H7 and N9 with a special emphasis on the avian H7N9 virus. Euro Surveill. 2014, 19, 20731. [CrossRef]

15. Hoffmann, B.; Harder, T.; Lange, E.; Kalthoff, D.; Reimann, I.; Grund, C.; Oehme, R.; Vahlenkamp, T.W.; Beer, M. New real-time reverse transcriptase polymerase chain reactions facilitate detection and differentiation of novel A/H1N1 influenza virus in porcine and human samples. Berl. Munch. Tierarztl. Wochenschr. 2010, 123, 286-292.

16. Hoffmann, B.; Hoffmann, D.; Henritzi, D.; Beer, M.; Harder, T.C. Riems influenza a typing array (RITA): An RT-qPCR-based low density array for subtyping avian and mammalian influenza a viruses. Sci. Rep. 2016, 6, 27211. [CrossRef]

17. Naguib, M.; Graaf, A.; Fortin, A.; Luttermann, C.; Wernery, U.; Amarin, N.; Hussein, A.H.; Sultan, H.; Al Adhadh, B.; Hassan, M.K.; et al. Novel real-time PCR-based patho- and phylotyping of potentially zoonotic avian influenza A subtype H5 viruses at risk of incursion into Europe in 2017. Euro Surveill. 2017, 22, 30435. [CrossRef]

18. Graaf, A.; Beer, M.; Harder, T.C. Real-time reverse transcription PCR-based sequencing-independent pathotyping of Eurasian avian influenza A viruses of subtype H7. Virol. J. 2017, 14, 137. [CrossRef]

19. Kearse, M.; Moir, R.; Wilson, A.; Stones-Havas, S.; Cheung, M.; Sturrock, S.; Buxton, S.; Cooper, A.; Markowitz, S.; Duran, C.; et al. Geneious Basic: An integrated and extendable desktop software platform for the organization and analysis of sequence data. Bioinformatics 2012, 28, 1647-1649. [CrossRef]

20. Katoh, K.; Standley, D.M. MAFFT multiple sequence alignment software version 7: Improvements in performance and usability. Mol. Biol. Evol. 2013, 30, 772-780. [CrossRef]

21. Larsson, A. AliView: A fast and lightweight alignment viewer and editor for large datasets. Bioinformatics 2014, 30, 3276-3278. [CrossRef]

22. Kibbe, W. OligoCalc: An online oligonucleotide properties calculator. Nucleic Acids Res. 2007, 35, W43-W46. [CrossRef]

23. Hassan, K.E.; El-Kady, M.F.; El-Sawah, A.A.A.; Luttermann, C.; Parvin, R.; Shany, S.; Beer, M.; Harder, T.C. Respiratory disease due to mixed viral infections in poultry flocks in Egypt between 2017 and 2018: Upsurge of highly pathogenic avian influenza virus subtype H5N8 since 2018. Transbound. Emerg. Dis. 2021, 68, 21-36. [CrossRef]

24. Naguib, M.M.; El-Kady, M.F.; Lüschow, D.; Hassan, K.E.; Arafa, A.-S.; El-Zanaty, A.; Hassan, M.K.; Hafez, H.M.; Grund, C.; Harder, T.C. New real time and conventional RT-PCRs for updated molecular diagnosis of infectious bronchitis virus infection (IBV) in chickens in Egypt associated with frequent co-infections with avian influenza and Newcastle Disease viruses. J. Virol. Methods 2017, 245, 19-27. [CrossRef]

25. Hoffmann, B.; Depner, K.; Schirrmeier, H.; Beer, M. A universal heterologous internal control system for duplex real-time RT-PCR assays used in a detection system for pestiviruses. J. Virol. Methods 2006, 136, 200-209. [CrossRef]

26. Abdelwhab, E.M.; Lüschow, D.; Harder, T.C.; Hafez, H.M. The use of FTA ${ }^{\circledR}$ filter papers for diagnosis of avian influenza virus. J. Virol. Methods 2011, 174, 120-122. [CrossRef]

27. Hassan, K.E.; Ali, A.; Dahshan, A.-H.M.; El-Sawah, A.A.; Shany, S.A.S.; El-Kady, M.F. Prevalence of avian respiratory viruses in broiler flocks in Egypt. Poult. Sci. 2016, 95, 1271-1280. [CrossRef]

28. Samy, A.; Naguib, M.M. Avian Respiratory Coinfection and Impact on Avian Influenza Pathogenicity in Domestic Poultry: Field and Experimental Findings. Vet. Sci. 2018, 5, 23. [CrossRef]

29. Shehata, A.A.; Sedeik, M.E.; Elbestawy, A.R.; Zain El-Abideen, M.A.; Kilany, W.H.; Ibrahim, H.H.; Ali, A. Co-infections, genetic, and antigenic relatedness of avian influenza H5N8 and H5N1 viruses in domestic and wild birds in Egypt. Poult. Sci. 2019, 98, 2371-2379. [CrossRef]

30. Liu, S.; Ji, K.; Chen, J.; Tai, D.; Jiang, W.; Hou, G.; Chen, J.; Li, J.; Huang, B. Panorama Phylogenetic Diversity and Distribution of Type A Influenza Virus. PLoS ONE 2009, 4, e5022. [CrossRef]

31. Zhuang, Q.; Wang, S.; Liu, S.; Hou, G.; Li, J.; Jiang, W.; Wang, K.; Peng, C.; Liu, D.; Guo, A.; et al. Diversity and distribution of type A influenza viruses: An updated panorama analysis based on protein sequences. Virol. J. 2019, 16, 85. [CrossRef]

32. Monne, I.; Ormelli, S.; Salviato, A.; De Battisti, C.; Bettini, F.; Salomoni, A.; Drago, A.; Zecchin, B.; Capua, I.; Cattoli, G. Development and validation of a one-step real-time PCR assay for simultaneous detection of subtype H5, H7, and H9 avian influenza viruses. J. Clin. Microbiol. 2008, 46, 1769-1773. [CrossRef]

33. Harfoot, R.; Webby, R. H5 influenza, a global update. J. Microbiol. 2017, 55, 196-203. [CrossRef]

34. King, J.; Harder, T.; Conraths, F.J.; Beer, M.; Pohlmann, A. The genetics of highly pathogenic avian influenza viruses of subtype H5 in Germany, 2006-2020. Transbound. Emerg. Dis. 2021, 68, 1136-1150. [CrossRef]

35. European Food Safety Authority; Adlhoch, C.; Fusaro, A.; Gonzales, J.; Kuiken, T.; Marangon, S.; Niqueux, E.; Staubach, C.; Terregino, C.; Aznar, I.; et al. Avian influenza overview February-May 2021. EFSA J. 2021, 19, 6951. 
36. Parvin, R.; Begum, J.A.; Chowdhury, E.H.; Islam, M.R.; Beer, M.; Harder, T.C. Co-subsistence of avian influenza virus subtypes of low and high pathogenicity in Bangladesh: Challenges for diagnosis, risk assessment and control. Sci. Rep. 2019, 9, 8306. [CrossRef]

37. Henritzi, D.; Zhao, N.; Starick, E.; Simon, G.; Krog, J.S.; Larsen, L.E.; Reid, S.M.; Brown, I.H.; Chiapponi, C.; Foni, E.; et al. Rapid detection and subtyping of European swine influenza viruses in porcine clinical samples by haemagglutinin- and neuraminidasespecific tetra- and triplex real-time RT-PCRs. Influenza Respir. Viruses 2016, 10, 504-517. [CrossRef] 